\title{
The sexist algorithm
}

\section{Melissa Hamilton}

University of Surrey School of Law, Guildford, United Kingdom

\section{Correspondence}

Melissa Hamilton, Senior Lecturer in Law \& Criminal Justice, University of Surrey School of Law, Guildford GU2 7XH, United Kingdom.

Email: melissa.hamilton@surrey.ac.uk

\begin{abstract}
Algorithmic risk assessment tools are informed by scientific research concerning which factors are predictive of recidivism and thus support the evidence-based practice movement in criminal justice. Automated assessments of individualized risk (low, medium, high) permit officials to make more effective management decisions. Computergenerated algorithms appear to be objective and neutral. But are these algorithms actually fair? The focus herein is on gender equity. Studies confirm that women typically have far lower recidivism rates than men. This differential raises the question of how well algorithmic outcomes fare in terms of predictive parity by gender.

This essay reports original research using a large dataset of offenders who were scored on the popular risk assessment tool COMPAS. Findings indicate that COMPAS performs reasonably well at discriminating between recidivists and non-recidivists for men and women. Nonetheless, COMPAS algorithmic outcomes systemically overclassify women in higher risk groupings. Multiple measures of algorithmic equity and predictive accuracy are provided to support the conclusion that this algorithm is sexist.
\end{abstract}

\section{1 | INTRODUCTION}

Across criminal justice systems, the "offender risk assessment enterprise has been moving at warp speed since the turn of the millennium and shows no signs of slowing.." ${ }^{11}$ The enterprise draws on the evidence-based practice movement in which factors shown by scientific studies to be predictive of recidivism inform management decisions. ${ }^{2}$ Officials seek such help as they operate in a high-stakes environment that requires weighing individual liberty, public safety, and limited resources.

Recent advances in statistical modeling mean that risk assessment practices are often automated. Algorithmic risk tools can draw upon big data to systematically produce individualized risk predictions. ${ }^{3}$ Proponents proclaim that 
predictive algorithms are inherently objective and neutral, thereby facilitating efficient and just decisions by criminal justice officials. ${ }^{4}$

Yet this guise of objectivity and neutrality has become the subject of heated debate. Recently, a coalition of over 100 groups self-described as comprising "civil and human rights, community, and data justice leaders from across the United States" (e.g., ACLU, NAACP, Electronic Frontier Foundation) signed onto "A Shared Statement of Civil Rights Concerns" expressing unease with the fairness of risk assessment tools used in criminal justice. ${ }^{5}$ The joint statement joins other voices in expressly calling for greater transparency and for third party audits of the tools and their underlying algorithms. ${ }^{6}$

A startling report in 2016 is an example of a third-party audit, and it notably uncovered bias. Investigative news journalists with ProPublica presented a series of statistical analyses involving a real-world dataset from Broward County, Florida, in which a popular risk tool named COMPAS was utilized in pretrial bail decisions. ${ }^{7}$ ProPublica concluded that COMPAS was racist in that its algorithm produced a much higher false positive rate for blacks than whites, meaning that it overpredicted high risk for blacks. ${ }^{8}$ ProPublica's publication attracted attention from media outlets. For example, NPR hosted one of its authors on a radio program titled, "The Hidden Discrimination in Criminal Risk-Assessment Scores." " Politico referred to the ProPublica report in its article "Is Your Software Racist?"10

COMPAS's corporate owner, Northpointe (since re-branded as equivant), quickly rejected such characterizations of its tool. ${ }^{11}$ After running their own statistical analyses on the same dataset ProPublica had compiled, Northpointe statisticians asserted that their results demonstrated that COMPAS outcomes achieved predictive parity for blacks and whites. ${ }^{12}$

It turns out a rather simple explanation accounts for the dispute: contrasting definitions of algorithmic fairness. ProPublica's estimation of false positive rates did not take into consideration that blacks had a higher recidivism rate than whites in the underlying dataset. In contrast, Northpointe's preferred metric of predictive parity fairly accounted for such base rate differences. Thus, the parties calculated distinctive statistics that, due to base rate differences, could not be consistent.

Nonetheless, in light of these issues, an equally important question is the possibility of a sexist algorithm. Studies confirm that women are far less likely than men to commit new crimes: a new meta-analysis concerning recidivism in the United States finds that male gender is significantly correlated with recidivism. ${ }^{13}$ Moreover, the meta-analysis reveals that gender is a stronger predictor of recidivism than other variables commonly included in recidivism risk tools. ${ }^{14}$ The obvious question, then, is whether algorithmic risk tools properly recognize this gender-based disparity.

This Article addresses this question with new statistical auditing of the COMPAS tool using the same Broward County dataset underlying the ProPublica and Northpointe studies. Section 2 briefly outlines the role of risk assessment in pretrial bail decisions. Section 3 reviews the evidence that algorithmic risk outcomes may not perform as well for women considering risk-relevant differences. Section 4 presents the study involving various statistical measures of algorithmic fairness. The gold standard method for examining the presence of group bias reveals that COMPAS exhibits gender test bias. Additional calculations regarding algorithmic fairness likewise show that COMPAS overclassifies females. COMPAS demonstrates gender bias even when using the algorithmic equity formula that Northpointe advocated in its debate with ProPublica. Conclusions follow.

\section{2 | PRETRIAL RISK ASSESSMENT}

Risk assessment in criminal justice is about predicting an individual's potential for criminal offending in the future. ${ }^{15}$ Risk assessment practices have typically advised back-end options (e.g., parole, post-incarceration supervision). ${ }^{16}$ Yet predictions about risk can inform in pretrial settings as well, such as determinations about pretrial release. ${ }^{17}$ To improve the fairness and efficacy of pretrial decisions, behavioral science experts encourage officials to rely upon the objective criteria in algorithmic risk tools. ${ }^{18}$ 
Legal reformers generally welcome this practice. Risk assessment serves the bail reform movement by offering a substitute to monetary bail. ${ }^{19}$ Risk-based judgments can alleviate harms that money bail systems cause poor and minority defendants, while increasing release rates of lower-risk defendants. ${ }^{20}$ The bail reform movement understands that early case decisions beget additional consequences. For example, pretrial detention correlates with increases in conviction rates, sentence length, and the likelihood of a sentence involving incarceration. ${ }^{21}$ Further, overclassifying pretrial defendants as "high risk" entails "gratuitous surveillance and overtreatment," which serves to increase their chances of failure. ${ }^{22}$

Notably, the United States Supreme Court in 1987 authorized pretrial detention based on a perception of a defendant's future dangerousness. ${ }^{23}$ The vagueness of "dangerousness" based largely on gut instinct has since then ceded to a more refined perspective of "risk assessment" with increasingly complex algorithms. ${ }^{24}$

While the rise in algorithmic risk assessment tools has earned praise in the evidence-based practices movement overall, some researchers are concerned with fairness and transparency. ${ }^{25}$ To the extent that justice decisions may bring negative consequences upon defendants, it is advisable to study whether and how an algorithmic tool disparately impacts protected groups, such as minorities or women. ${ }^{26}$

\section{3 | THE POTENTIAL FOR A SEXIST ALGORITHM}

The empirical study reported herein focuses on gender disparities for important reasons. Criminal justice critics concentrate much anti-discrimination attention on race/ethnicity, often relegating gender bias as a lesser concern. For example, the Shared Statement of Civil Rights Concerns signed by the 100 plus groups is deeply troubled by the potential for algorithmic bias in risk tools and underscores racial inequities numerous times; it passingly mentions the potential for gender bias. ${ }^{27}$ Notwithstanding, algorithmic unfairness for women is perhaps just as likely considering that women consistently have lower recidivism rates than men. ${ }^{28}$ Unless a risk tool accounts for these base rate differences, overclassification of females is a foreseeable result, as is differential validity-meaning that the tool will not predict at equal levels of accuracy in both gender groups. ${ }^{29}$ Further, observers note that too few studies exist that directly examine the impact of gender on the predictive ability of risk assessment tools. ${ }^{30}$ This research can help fill that gap.

Certain criminological literature provides some explanation for why recidivism prediction tools might perform poorly for women. Most risk assessment tools contain what are purported to be "gender-neutral" factors such as criminal history, age, mental health, and substance abuse. ${ }^{31}$ Researchers have objected that these "gender-neutral" factors may not be as evenhanded as might first appear to be the case, in that many of these factors result in higher correlations to recidivism for men. ${ }^{32}$ In contrast to such "gender-neutral" predictors, research indicates that certain gender-responsive factors correlate with better outcomes specifically for women: examples include items relating to personal relationship troubles, victimization, effects of trauma, and parenting stress. ${ }^{33}$ The (relatively sparse) research on the subject suggests that incorporating these kinds of gender-responsive factors into risk assessment tools targeting women may achieve greater predictive abilities for them as a result. ${ }^{34}$

From legal and ethical perspectives, whether gender-directly or by proxy via gender-responsive factors-may be incorporated into risk tools remains largely unresolved. Scholars at times contend that gender is a protected grouping and therefore should be given no significance in criminal justice decisionmaking. ${ }^{35}$ Bernard Harcourt would call gender-cognizant risk assessment unacceptable gender profiling. ${ }^{36}$ Nonetheless, it seems simplistic to assume that fairness requires removing all vestiges of protected classes.

Algorithms that are designed to be neutral regarding protected groups may still produce disparate impact. ${ }^{37}$ If an algorithm's training data imbedded discriminatory practices, such as inequitable police decisions to arrest, then its outcomes may replicate those same biases. ${ }^{38}$

At least one legal scholar specifically advocates incorporating gender-based risk assessment in criminal justice decisions expressly because gender is shown to be a statistically significant predictor of reoffending. ${ }^{39}$ Indeed, the inclusion of gender in a tool would appear to positively serve legal and empirical interests if it favorably improves 
predictive accuracy, thus permitting the tool to better inform management decisions. ${ }^{40}$ For this very reason, the American Law Institute's new Model Penal Code expressly advocates the use of gender in risk assessment to inform sentencing decisions because of its relevance to protecting public safety. ${ }^{41}$

Statisticians associated with COMPAS likewise have long acknowledged that gender-specific calibrations for risk factors are justified considering risk-relevant differences. ${ }^{42}$ Northpointe therefore offers as an option COMPAS risk scales with separate male and female norming, in which decile scores vary based on which gendered norm group applies. ${ }^{43}$ Broward County (the site of the study here), though, elects not use the gender-specific norms. ${ }^{44}$ In contrast, officials in other states embrace gender-responsivity factors that better reflect the risk factors unique to women. Pennsylvania, for example, directly incorporates gendered items into the risk assessment tool it has created for sentencing. ${ }^{45}$ The state of Wisconsin uses the gender-responsive version of COMPAS. ${ }^{46}$

A recent case opinion on point is of some assistance. The Wisconsin Supreme Court addressed the use of the COMPAS gendered scales in sentencing decisions and, in denying a due process challenge, noted that not including gender would lead to misclassifying both sexes. ${ }^{47}$ This case does not establish any strong precedent, however, as it currently stands as an outlier. As the United States Solicitor General contended in an amicus curiae brief upon petition for a writ of certiorari before the United States Supreme Court, the legal challenge to gender and COMPAS is premature as "due process and other constitutional issues have not yet been fully aired in state courts and lower federal courts." 48 The high court thereafter denied cert. ${ }^{49}$

\section{4 | ALGORITHMIC GENDER BIAS}

This section reports on a study of COMPAS, a popular algorithmic risk assessment tool, ${ }^{50}$ employing the same realworld dataset as ProPublica compiled for its evaluation of outcomes for blacks versus whites. ${ }^{51}$ The data and the tool will be briefly addressed. Then the statistical results are provided. Recent attention underscores the importance of algorithmic fairness, whereby bias in risk assessment tool outcomes can result in disparate impact on protected groups. $^{52}$ This comprises an inherently interdisciplinary project, as data scientists may not be fully cognizant of civil rights issues, while legal practitioners often lack statistical skills. Further, "[i]t is important to bear in mind that fairness itself-along with the notion of disparate impact-is a social and ethical concept, not a statistical concept." ${ }^{23}$ Nonetheless, multiple measures of accuracy and fairness exist in the algorithmic risk literature. Although it is generally impossible for any risk tool to comply with them all, ${ }^{54}$ the results that follow draw upon an array of such measures to provide a layered perspective on the gender-based abilities of COMPAS that has not been not offered elsewhere.

\subsection{Introducing the study}

The primary dataset includes 6,172 individuals arrested in Broward County, Florida, and scored on the COMPAS general recidivism risk scale soon after their arrests in 2013 and $2014 .^{55}$ The pretrial services division of the Broward County Sheriff's Office has been using COMPAS since 2008 to inform judicial determinations concerning pretrial release. ${ }^{56}$ The study used a two-year recidivism follow-up period. These analyses generally followed the methodology of the ProPublica researchers in terms of defining what acts comprise general or violent recidivism, excluding cases with missing data, and excluding cases where the individuals were not scored on COMPAS in a timely manner. ${ }^{57}$

COMPAS is the acronym for Correctional Offender Management Profiling for Alternative Sanctions. Northpointe, COMPAS's owner, claims that the instrument is protected by trade secret law and thus declines to reveal its algorithm, which is likely a reason why ProPublica was interested in auditing the tool. The COMPAS general recidivism risk scale contains about two dozen items related to age, criminal history, drug problems, and vocational/educational problems (e.g., grades, suspensions, employment). ${ }^{58}$ The COMPAS algorithm produces 
outcomes as decile scores of 1-10, with higher deciles representing greater predicted risk. COMPAS then subdivides decile scores into three, ordinal risk bins: low risk (deciles 1-4); medium risk (deciles 5-7), and high risk (deciles 8-10).

\subsection{The validity of risk bins}

Scientific evaluation of the validity of predictive algorithms differentiates the concepts of discrimination and calibration. Discrimination concerns relative accuracy, here in terms of how well the tool distinguishes recidivists from nonrecidivists. ${ }^{59}$ In contrast, calibration concerns absolute predictive accuracy. ${ }^{60}$ Discrimination and calibration each offers a distinct contribution. For instance, a "scale that ranks well, but systematically overestimates or underestimates risk might have good discriminative properties but be poorly calibrated to the population under examination."61

We can begin the analysis of the tool's discrimination and calibration abilities with a simple graph. Figure 1 charts the recidivism rate of offenders assigned to each COMPAS risk bin, separated by gender.

In each risk bin, females recidivated at lower rates than males (between 9 and 12\% lower), and the differences were statistically significant $(p<.01)$. Figure 1 , thereby, visually demonstrates that COMPAS overclassifies women as compared with men. Medium and high risk classifications simply do not have the same predictive utility across genders.

These results should not be surprising to Broward County officials or Northpointe. A 2010 validation study commissioned by Broward County officials expressly showed that, consistently with this study's results in Figure 1 for general recidivism, across risk bins males recidivated at far higher rates than females classified in the same bins. ${ }^{62}$ Oddly, the independent academic researchers conducting the validation did not, at least in the publicly available document, recommend that the agency begin to use the gendered norms to improve calibration accuracy. Despite the findings in 2010, Broward County has continued to use COMPAS scales that are not gender normed, and thus the same gender-biased results are shown here.

These variances, though, pale in comparison to the discrepancies for the COMPAS violent recidivism scale. COMPAS offers a separate risk tool for violent recidivism. In lieu of the more general criminal history scale and drug problem scale that the general recidivism tool uses, the violence risk tool depends on items regarding history of violence

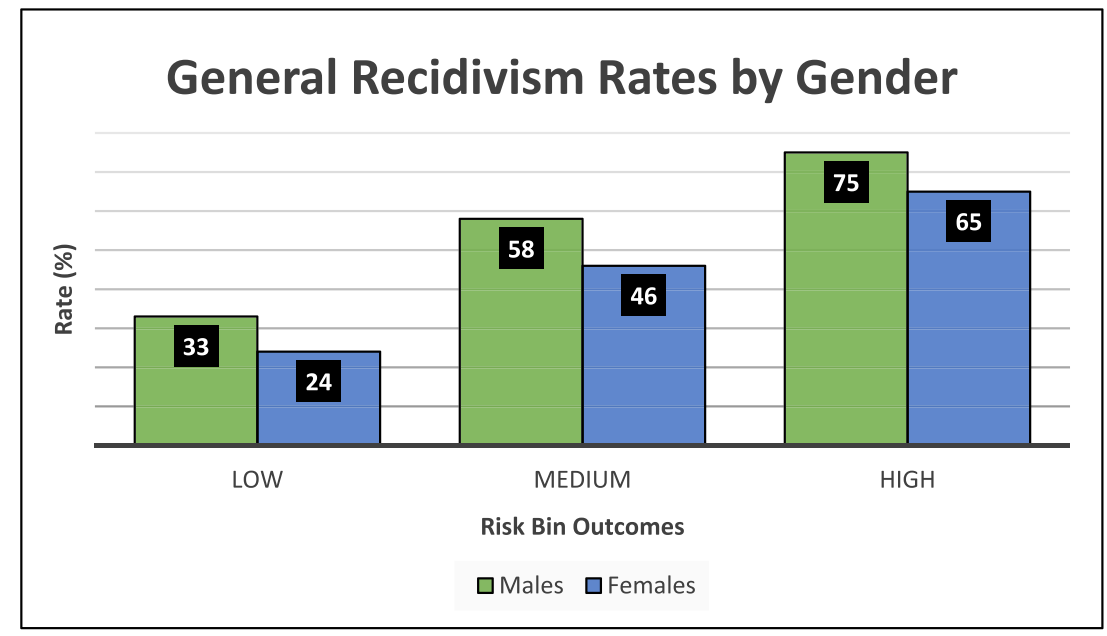

$n=6,172$

FIGURE 1 General recidivism rates [Colour figure can be viewed at wileyonlinelibrary.com] 
and of non-compliance (e.g., parole revocation). Though the violence risk tool is not the focus in this article, a visual of its ability still can help make the point that gender matters to risk assessment. Figure 2 accordingly presents violent recidivism rates by gender according to the risk bins assigned by the COMPAS violence risk tool.

At the extreme in the high risk category, males violently reoffended at a rate more than twice the rate of females. As systematic overclassification in any group presents evidence of bias, ${ }^{63}$ Figures 1 and 2 make clear that the general and violent recidivism tools are biased against women as a group. These figures also highlight the contrast between discrimination and calibration previously mentioned. COMPAS risk binning appears to have discriminatory ability in that higher risk bins are related to increased recidivism rates for both genders. However, its calibration shows inequalities between genders, i.e., differential calibration.

\section{3 | Test bias}

An alternative, more complex statistical design exists for third-party auditing of bias. Researchers investigating the existence of group bias in psychological testing have settled on a best practice methodology. This gold standard involves a series of nested models of regression equations involving the test, the group(s) of interest, and an interaction term (test*group) as predictors of test outcomes. ${ }^{64}$ The regression evaluates the linear relationship between one or more predictors with a response (outcome) variable. ${ }^{65}$ The interaction term examines the product of the predictor variables (test and group) to determine whether the effect on the outcome of either predictor is moderated by the presence of the other. ${ }^{66}$

This nested model method detects group differences in the form of the relationship between the test and the outcome in terms of the intercept and slope ${ }^{67}$ to reveal differential prediction-that is, the potential for unequal predictive ability. ${ }^{68}$ The rule of thumb in the psychological assessment field is that a significant group difference in either the intercept or the slope indicates that a single regression equation for the groups combined will predict inaccurately for one or both groups, and therefore a separate equation for each group must be considered. ${ }^{69}$ Unequal intercepts or slopes also signify disparate impact; notably, disparate impact can exist without evidence of discriminatory intent. ${ }^{70}$ Enterprising criminal justice researchers have recently applied this methodological practice to evaluating group bias in risk assessment tools. ${ }^{71}$

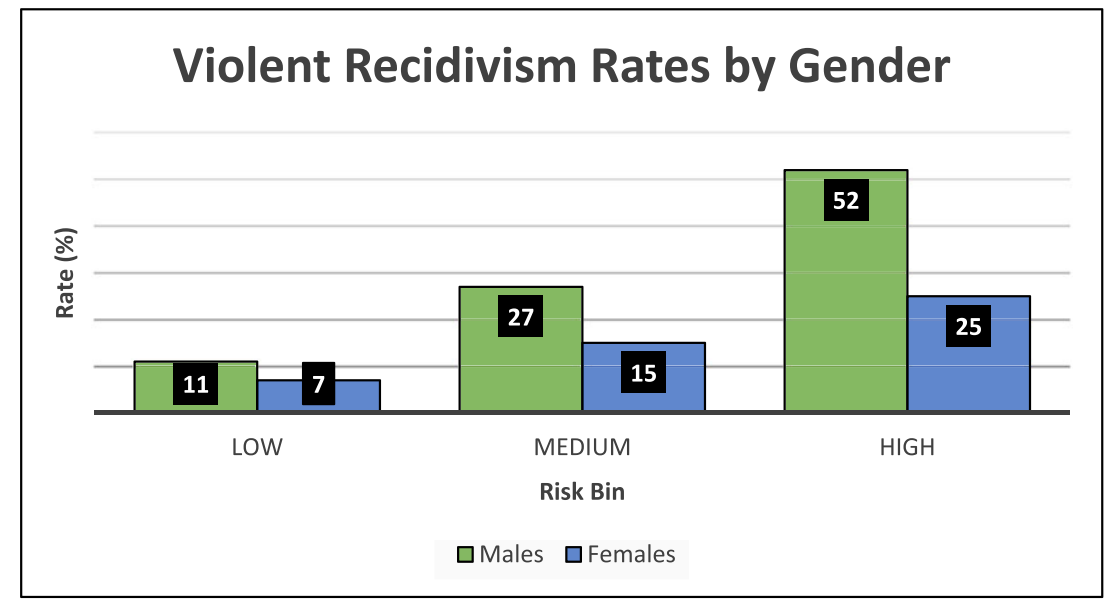

$n=4,020$

FIGURE 2 Violent recidivism rates [Colour figure can be viewed at wileyonlinelibrary.com] 
Table 1 presents the relevant regression equation results across nested models. In compliance with the gold standard for assessing test bias, the nested model structure here utilized variables labeled as Female (coded as female $=1$, male $=0$ ) and COMPAS decile score, and an interaction between them as Female*COMPAS decile score. A fourmodel structure is employed with the outcome variable being recidivism. Model 1 tests only the female variable; Model 2 tests just COMPAS decile score; Model 3 includes the Female and COMPAS decile score variables; and Model 4 retains Female and COMPAS decile score while adding the interaction term. It is noted that logistic regression is the appropriate method when the outcome is dichotomous in nature (e.g., recidivist = yes/no). The regression coefficients are translated in the table into odds ratios for interpretive purposes.

Models 1-4 in Table 1 indicate that gender and decile score are significantly predictive of general recidivism both individually and when entered together. The odds of women recidivating (across the models) are approximately .6 times that of men (i.e., the odds of women reoffending are about $40 \%$ less than the odds of men reoffending). ${ }^{72}$ The odds ratios for decile score (across models) indicate that, for every one unit increase in decile score, the odds of recidivism increase by $32 \%$. This latter finding supports the predictive ability of COMPAS generally for males and females.

Gender is statistically significant in Model 3, which means that the regression lines for males and females do not share the same intercepts. As the odds for Female are less than one, men are more likely to reoffend even when controlling for COMPAS decile score. Model 4 shows that the interaction term, however, is not statistically significant. This latter finding indicates that the form of the relationship between (a) gender and general recidivism is not moderated by the decile score, and (b) decile score and general recidivism is not moderated by gender. In other words, these findings indicate that gender and decile score each have independent and significant effects on general recidivism.

In sum, the set of nested models demonstrates that the bias is in the intercept (men have a higher recidivism rate) but not in the slope of the relationship (because a higher decile score is associated with a similar increase in recidivism rates for both genders). Bias in the intercept here shows that the issue is overclassification for women and confirms test bias and disparate impact. On the other hand, a lack of bias in the slope is further support that COMPAS has good discriminatory ability for each gender.

One may reasonably wonder if such gender-biased results are due to the few variables in these models. Additional analysis tends to refute this possibility. In a more robust model (not presented herein), the gender variable retained statistical significance and in the same direction (i.e., a negative predictor of general recidivism for women) when also controlling for decile, race, age, criminal history, and charge degree. ${ }^{73}$

\section{4 | Equal calibration}

A related definition of algorithmic fairness is known as equal calibration. It requires that expected rates of recidivism be the same as observed recidivism rates in action, and that this is the case irrespective of group membership. ${ }^{74}$

TABLE 1 Logistic regressions predicting general recidivism

\begin{tabular}{lccrr} 
Predictor & Model 1 & Model 2 & Model 3 & Model 4 \\
\hline Female & $.588^{*}$ & - & $.623^{*}$ & $.624^{*}$ \\
Decile & - & $1.323^{*}$ & $1.321^{*}$ & $1.321^{*}$ \\
\hline Female*decile interaction & - & - & - & .999 \\
Constant & .921 & .239 & $7,606.26$ & .263 \\
-2 LL & $8,442.56$ & $7,650.88$ & 900.15 & $7,606.26$ \\
$x^{2}$ & 63.86 & 855.53 & & 900.15 \\
$n=6,172$ & & & & 996 \\
\hline
\end{tabular}

Notes:

${ }^{*} p<.001$. Values for the variables represent odds ratios. 
Figure 3 presents a graph involving three lines: a single expected recidivism rate line (applicable to both genders) and then (actual) observed rates of recidivism in the Broward County dataset by each gender. The expected general recidivism rates by decile were calculated from Model 2 statistics in Table 1.

Figure 3 exemplifies several areas of concern about calibration and group bias. Observed recidivism rates fail to consistently increase in a linear fashion as decile scores increase, though the deviations are not dramatic. Hence, there are some weaknesses in terms of discriminatory ability between groupings in certain adjacent decile scores. Contrast these results with those in Figure 1. In Figure 1 the use of the three risk bins showed good discrimination in that the rates of reoffending in each bin significantly increase from low to medium to high. However, when decile scores are used, as exemplified in Figure 3, the discriminatory ability is partially discredited, as observed recidivism rates fail to measurably increase across each decile increment for either gender.

The graphic in Figure 3 further visually illustrates calibration errors. Perfect calibration requires that observed rates equal expected rates. ${ }^{75}$ The spread (i.e., gaps) between datapoints of observed rates (for each gender) and the expected recidivism line represents these errors. Observed recidivism rates for males more closely track the unitary expected rate line. Also, males recidivated at higher rates than females at every decile score, ranging from a variance of $4 \%$ (Decile 1) to $18 \%$ (Decile 2), with an average of $11 \%$. Overall, calibration errors against women exist at every decile and in the same direction. The observed recidivism rates for females were significantly lower than expected rates at every score, ranging from $4 \%$ (Decile 3) to $12 \%$ (Decile 8 ), with an average of $9 \%$. In sum, COMPAS overpredicts risk for females at every decile score, thereby indicating systemic gender bias.

\section{5 | Misclassification errors}

The algorithmic science literature breaks discrimination and calibration down into other, more specific definitional measures. Two were chosen herein as they are at the heart of the ProPublica and Northpointe dispute.

One conceptualization of algorithmic fairness is referred to as error rate balance. It determines whether equal group error rates exist, for example in the form of the false positive rate (FPR). ${ }^{76}$ The FPR is not about forecasting accuracy, as it is retrospective in nature. ${ }^{77}$ The FPR is the percentage of known non-recidivists who were incorrectly

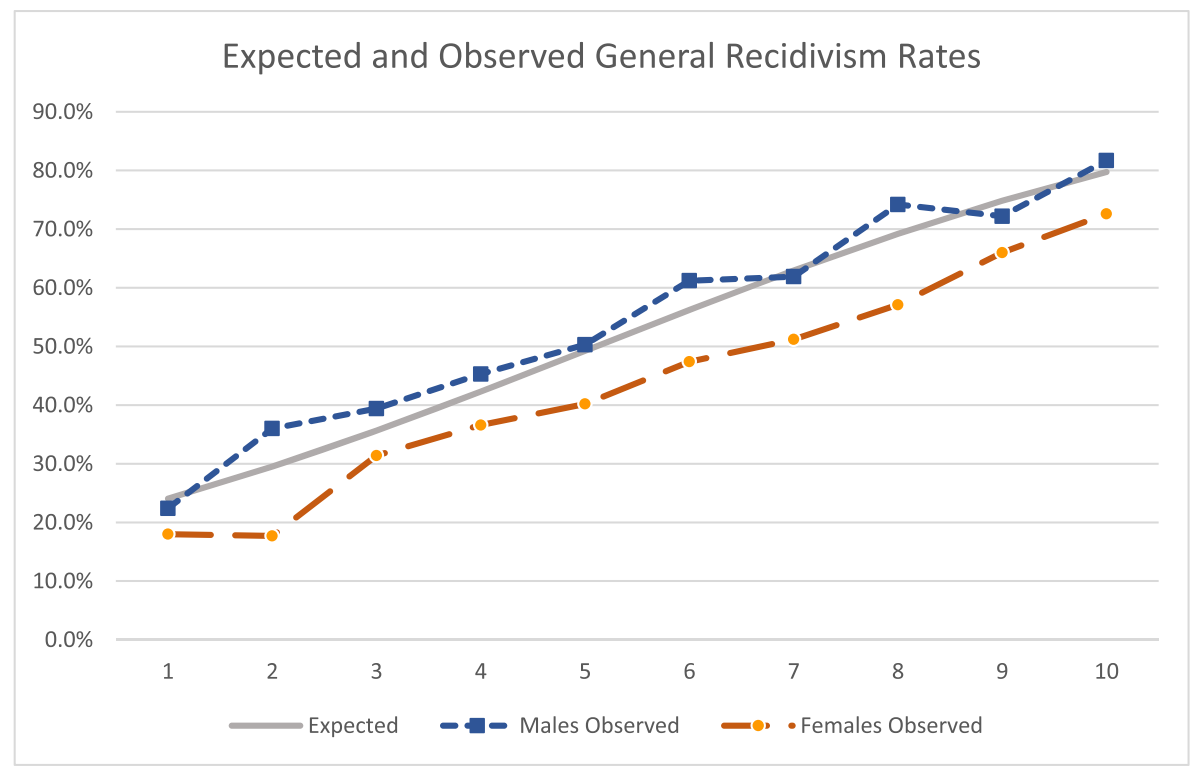

FIGURE 3 Equal calibration graphing [Colour figure can be viewed at wileyonlinelibrary.com] 
classified by the algorithm as high risk (predicted to reoffend). ${ }^{78}$ Predictive parity is another popular definition for algorithmic fairness, which requires similar positive predictive values (PPVs) between groups. ${ }^{79}$ Unlike the FPR, the PPV assesses calibration accuracy, is prospective in nature, and is highly influenced by the base rate. The PPV signifies the proportion of persons the algorithm classified as high risk who then reoffended. ${ }^{80}$

The FPR and PPV require that the sample be divided into just two groupings: one representing individuals predicted to be recidivists and the other non-recidivists. Regarding COMPAS, researchers typically opt to contrast the low risk bin with a combined medium/high risk grouping (the lower cutpoint) or merge the low/medium risk bins together with compare to the high risk category (the higher cutpoint). ${ }^{81}$ Table 2 provides statistics for FPRs and PPVs and offers both cutpoints, contrasting by gender.

From Table 2, at the lower cutpoint (low versus medium/high) the FPRs are the same, but at the higher cutpoint males have a higher FPR than females ( $9 \%$ versus $7 \%$, respectively). This suggests a bias against men, with a higher percentage of false positives in the high risk bin, though a $z$-test indicates that the difference is not statistically significant. However, Table 2 indicates statistically significant differences in PPVs (from z-tests). Males are associated with higher PPVs at each cutpoint, meaning that predictions of recidivism were correct more often for men and thus the fairness notion of predictive parity for gender is not met.

In sum, these additional measures of algorithmic fairness again show that COMPAS systematically overclassifies women. These findings provide additional support for differential validity and that COMPAS does not meet the algorithmic fairness standard of predictive parity.

Notice that the PPV measures indicate bias against females while the FPRs do not. This contrast helps explains the dispute between Northpointe and ProPublica as to whether COMPAS was racist. The results there were the opposite, with the PPVs highlighted by Northpointe indicating no bias, while the FPRs used by ProPublica showed bias. Northpointe there contended that the PPV is what "should be analyzed if one is interested in testing for racial bias." ${ }^{82}$ Hence, using Northpointe's preferred statistic, the results in Table 2 reveal that COMPAS outcomes have a gender bias against women.

\subsection{Area under the curve}

The final analysis herein involves a metric called the area under the curve (AUC)-it is derived from a statistical plotting of true positives and false positives across a risk tool's rating system. ${ }^{83}$ More specifically, an AUC is a discrimination index that represents the probability that a randomly selected recidivist received a higher risk classification than a randomly selected non-recidivist. ${ }^{84}$ AUCs range from 0 to 1.0, with 0.5 indicating no better accuracy than chance and a 1.0 meaning perfect accuracy. ${ }^{85}$

The AUCs using COMPAS decile scores were .711 for males and .697 for females, a difference that is not statistically significant. This indicates that COMPAS shows comparable ability to discriminate recidivists from nonrecidivists for both genders, which is consistent with the graphic display in Figure 1. Still, as discussed elsewhere, the AUC has serious limitations, and thus cannot present a holistic portrait of a tool's abilities. ${ }^{86}$ The AUC is too

TABLE 2 Measures of discrimination and calibration

\begin{tabular}{|c|c|c|c|c|}
\hline \multirow{2}{*}{ Measure } & \multicolumn{2}{|c|}{ Low v. medium/high } & \multicolumn{2}{|c|}{ Low/medium v. high } \\
\hline & Males & Females & Males & Females \\
\hline \multicolumn{5}{|c|}{ Discrimination } \\
\hline FPR & .30 & .30 & .09 & .07 \\
\hline \multicolumn{5}{|l|}{ Calibration } \\
\hline PPV & .65 & $.52^{*}$ & .75 & $.65^{*}$ \\
\hline
\end{tabular}

Note:

${ }^{*} p<.001$. 
commonly misinterpreted as measuring calibration accuracy, but in fact a higher AUC does not mean more accurate prospective prediction. ${ }^{87}$ Further, the AUC cannot calculate how well an instrument selects those at high risk. ${ }^{88}$ For example, the AUC could be very high even if no recidivists were ranked as high risk. To use a hypothetical, the AUC for COMPAS would reflect perfect accuracy $(A \cup C=1.0)$ where all recidivists were classified Decile 2 and all nonrecidivists as Decile 1 (i.e., all were classified as low risk), with very little distinction considering the decile scale ranges from 1 to 10 .

\section{7 | Study limitations}

Several limitations should be mentioned. The single site limits generalization of results. This study relied upon archival data, and it is thereby possible for there to have been systemic errors in data collection that are not observable. Recidivism outcomes were from official records and thus will not include undetected crimes. The dataset did not include interrater reliability scores that would confirm the dependability of COMPAS scoring across evaluators and over time. Further, it would have been preferable to control for aspects of supervision, as pretrial services/conditions may moderate reoffending rates, but secondary data analysis did not permit it.

\section{5 | CONCLUSION}

This study shows strong evidence of systematic gender bias in a popular risk tool in terms of failing on multiple algorithmic fairness definitions aimed at calculating calibration equity. To COMPAS's credit, its owners realize this and offer gender-specific norms. When agencies, such as the one studied here, decline to incorporate gendered scoring, it is unsurprising that risk outcomes will present disparate impact on women. The unfortunate consequence is that the risk tool overclassifies women and thus more of them are likely to be unfairly treated in criminal justice decisions and be subject to unnecessary levels of supervision.

It remains an open question as to whether gender can expressly be included in risk tools from a legal perspective. What is clear is that not incorporating gender is unjustified from an empirical perspective, as it critically undermines basic requirements of the evidence-based practice movement.

\section{ENDNOTES}

1」. Stephen Wormith, Automated Offender Risk Assessment, 16 CRIMINOLOGY \& PUB. POL'Y 281, 281 (2017).

${ }^{2}$ Kelly Hannah-Moffat, Algorithmic Risk Governance: Big Data Analytics, Race and Information Activism in Criminal Justice Debates, THEORETICAL CRIMINOLOGY (forthcoming 2019), http://journals.sagepub.com/doi/pdf/10.1177/1362480618763582.

${ }^{3}$ Angéle Christin, Algorithms in Practice: Comparing Web Journalism and Criminal Justice, BIG DATA \& Soc'Y 1, 2 (July-Dec. 2017), http://journals.sagepub.com/doi/pdf/10.1177/2053951717718855.

${ }^{4}$ Arthur Rizer \& Caleb Watney, Artificial Intelligence can Make our Jail System More Efficient, Equitable and Just, 23 Tex. Rev. L. \& Pol. 181, 183 (2019).

${ }^{5}$ African American Ministers in Action et al., The Use of Pretrial "Risk Assessment" Instruments: A Shared Statement of Civil Rights Concerns 2 (2018), http://civilrightsdocs.info/pdf/criminal-justice/Pretrial-Risk-Assessment-Full.pdf [hereinafter Shared Statement].

${ }^{6}$ Id. at 7; Robyn CAPLAN et Al., Algorithmic Accountability: A PrIMER 10 (Apr. 18, 2018), https://datasociety.net/pubs/ alg_accountability.pdf.

7Julia Angwin et al., Machine Bias, PRoPublicA (May 23, 2016), https://www.propublica.org/article/machine-bias-riskassessments-in-criminal-sentencing.

${ }^{8}$ Id.

${ }^{9}$ NPR, The Hidden Discrimination in Criminal Risk-Assessment Scores (May 24, 2016), https://www.npr.org/2016/05/24/ 479349654/the-hidden-discrimination-in-criminal-risk-assessment-scores.

${ }^{10}$ Li Zhou, Is Your Software Racist?, PoLitico (Feb. 7, 2018, 5:05 AM), https://www.politico.com/agenda/story/2018/02/07/ algorithmic-bias-software-recommendations-000631. 
${ }^{11}$ William Dieterich et al., COMPAS Risk Scales: Demonstrating Accuracy Equity and Predictive Parity 2 (July 8, 2016 ), http://go. volarisgroup.com/rs/430-MBX-989/images/ProPublica_Commentary_Final_070616.pdf.

${ }^{12}$ Id.

${ }^{13}$ Antonis Katsiyannis et al., Adult Recidivism in the United States: A Meta-Analysis 1994-2015, 27 J. CHILD \& FAMILY STUD. 686, 691 (2018).

${ }^{14}$ Id.

${ }^{15}$ Melissa Hamilton, Risk-Needs Assessment: Constitutional and Ethical Challenges, 52 AM. CRIM. L. REV. 231,232 (2015).

${ }^{16}$ Jessica M. Eaglin, Constructing Recidivism Risk, 67 EMORY L.J. 59, 67 (2017).

${ }^{17}$ Kristin Bechtel et al., A Meta-Analytic Review of Pretrial Research: Risk Assessment, Bond Type, and Interventions, 42 AM. J. CRIM. JUST. 443, 444 (2017).

${ }^{18}$ Rizer \& Watney, supra note 4 , at 186.

${ }^{19}$ Megan Stevenson, Assessing Risk Assessment, 102 MINN. L. REV. (forthcoming 2019), https://papers.ssrn.com/sol3/Delivery.cfm/SSRN_ID3139944_code2420348.pdf?abstractid $=3016088 \&$ mirid $=1$.

${ }^{20}$ John Logan Koepke \& David G. Robinson, Danger Ahead: Risk Assessment and the Future of Bail Reform, 93 Wash. L. Rev. 1725, 1725 (2018).

${ }^{21}$ James C. Oleson et al., The Sentencing Consequences of Federal Pretrial Supervision, 63 CRIME \& DELINQ. 313, 316-17 (2017) (citing studies).

${ }^{22}$ Whitney Threadcraft-Walker et al., Gender, Race/Ethnicity and Prediction: Risk in Behavioral Assessment, 54 J. CRIM. JUST. 12, 13 (2018).

${ }^{23}$ United States v. Salerno, 481 U.S. 739, 751 (1987).

${ }^{24}$ Kirk Heilbrun, Evaluation for Risk of Violence in Adults 708 (2009).

${ }^{25}$ Flavio Chierichetti et al., Fair Clustering Through Fairlets, 31st Conference on Neural Information Processing Systems 1 (2017), http://www.chierichetti.name/papers/fairlets.pdf.

${ }^{26}$ Mark MacCarthy, Standards of Fairness for Disparate Impact Assessment of Big Data Algorithms, 48 CUMBERLAND L. REV. 67, 67 (2017).

${ }^{27}$ Shared Statement, supra note 5 , at 9.

${ }^{28}$ Katsiyannis et al., supra note 13 , at 691.

${ }^{29}$ Julia A. Yesberg et al., Predicting Women's Recidivism: Validating a Dynamic Community-Based "Gender-Neutral" Tool, 62 PROB. J. 33, 36 (2015).

${ }^{30}$ Threadcraft-Walker et al., supra note 22 , at 12.

${ }^{31}$ Patricia Van Voorhis et al., Women's Risk Factors and Their Contributions to Existing Risk/Needs Assessment, 37 CRIM. JUST. \& BEHAV. 261, 262 (2010).

${ }^{32}$ VAlerie R. BeLL, Gender-Responsive Risk Assessment In CORRECTIONS 108 (2014).

${ }^{33}$ Van Voorhis et al., supra note 31, at 262.

${ }^{34}$ See generally Krista S. Gehring \& Patricia Van Voorhis, Needs and Pretrial Failure, Additional Risk Factors for Female and Male Pretrial Defendants, 41 CRIM. JUST. \& BEHAV. 943 (2014).

${ }^{35}$ Jennifer Skeem et al., Gender, Risk, Assessment, and Sanctioning: The Cost of Treating Women Like Men, 40 LAW \& HuM. BEHAV. 580, 582 (2016) (discussing both sides).

${ }^{36}$ Bernard Harcourt, Against Prediction 227 (2007).

${ }^{37}$ Frank Pasquale, Toward a Fourth Law of Robotics, 78 OHIO ST. L. J. 1243, 1247 (2017).

${ }^{38}$ Anupam Chander, The Racist Algorithm?, 115 MICH. L. REV. 1023, 1036 (2017).

${ }^{39}$ Richard G. Kopf, Federal Supervised Release and Actuarial Data (Including Age, Race, and Gender), 27 FED. SENT'G REP. 207, 211 (2015).

${ }^{40}$ Christopher Slobogin, Principles of Risk Assessment, 15 OHIO ST. J. CRIM. L. 583, 592 (2018).

${ }^{41}$ Am. L. Inst., Model Penal Code: Sentencing: Proposed Final Draft §7.03(4)(b) (2017).

${ }^{42}$ Tim Brennan et al., Evaluating the Predictive Validity of the COMPAS Risk and Needs Assessment System, 36 CRIM. JUST. \& BeHAV. 21, 24 (2009).

${ }^{43}$ Id. at 25. 
${ }^{44}$ Blomberg et Al., VAlidation OF The COMPAS Risk Assessment Classification InStrument 13 (2010).

${ }^{45}$ Penn. Comm'n Sentencing, Policy Overview of the Risk Assessment Instrument (2018), http://pcs.la.psu.edu/guidelines/ proposed-risk-assessment-instrument/additional-information-about-the-proposed-sentence-risk-assessment-instrument/ policy-overview-of-the-sentence-risk-assessment-instrument/view.

${ }^{46}$ Wisc. Dept. Corrections, Becky Young Community Corrections Recidivism Reduction Fiscal Year 2017 Report 4 (Sept. 2017), https://doc.wi.gov/Documents/AboutDOC/Reentry/2017BeckyYoungReport.pdf.

${ }^{47}$ State v. Loomis, 881 N.W.2d 749, 766 (Wisc. 2016).

${ }^{48}$ Brief for the United States as Amicus Curiae at 21, Loomis v. Wisconsin, 137 S. Ct. 1240 (2017) (No. 16-6387), http:// www.scotusblog.com/wp-content/uploads/2017/05/16-6387-CVSG-Loomis-AC-Pet.pdf.

49137 S. Ct. 2290 (2017).

${ }^{50}$ EQUIVANT, COMPAS CLASSIFICATION 2 (2017), http://equivant.volarisgroup.com/assets/img/content/Classification.pdf.

${ }^{51}$ COMPAS is the "most successful and popular application of machine learning." Wormith, supra note 1, at 285. (citing developers using a decision tree model to educate the tool).

${ }^{52}$ MacCarthy, supra note 26 , at 67.

${ }^{53}$ Alexandria Chouldechova, Fair Prediction with Disparate Impact: A Study of Bias in Recidivism Prediction Instruments, 5 BIG DATA 153, 154 (2017).

${ }^{54}$ Richard Berk et al., Fairness in Criminal Justice Risk Assessments: The State of the Art, SOCIOLOGICAL METHODS \& RES. (forthcoming 2018), https://journals.sagepub.com/doi/pdf/10.1177/0049124118782533.

${ }^{55}$ ProPublica obtained the underlying raw data via Freedom of Information Act requests and has generously made it available for other researchers to access here: https://github.com/propublica/compas-analysis.

${ }^{56}$ BLOMBERG ET AL., supra note 44, at 15-16.

${ }^{57}$ See generally Jeff Larson et al., How We Analyzed the COMPAS Recidivism Algorithm, ProPuBLICA (May 23, 2016), https:// www.propublica.org/article/how-we-analyzed-the-compas-recidivism-algorithm.

${ }^{58}$ NORTHPOINTE, COMPAS CORE NORMS FOR AdULT INSTITUTIONS 80 tbl. 3.41 (Feb. 11, 2014), https://epic.org/algorithmictransparency/crim-justice/EPIC-16-06-23-WI-FOIA-201600805-WIDOC_DAI_norm_report021114.pdf.

${ }^{59}$ L. Maaike Helmus \& Kelly M. Babchishin, Primer on Risk Assessment and the Statistics Used to Evaluate its Accuracy, 44 CRIM. JUST. \& BEHAV. 8, 11 (2017).

${ }^{60}$ Id.

${ }^{61}$ Philip D. Howard, The Effect of Sample Heterogeneity and Risk Categorization on Area Under the Curve Predictive Validity Metrics, 44 CRIM. JUST. \& BEHAV. 103, 105 (2017).

${ }^{62}$ Id. at 38 tbl. 4 tf.

${ }^{63}$ Stevenson, supra note 19.

${ }^{64}$ Jeanne A. Teresi \& Richard N. Jones, Bias in Psychological Assessment and Other Measures, in APA HANDBOOK OF TESTING AND Assessment IN PSYCHOlogy 139, 144 (vol. 1 2013).

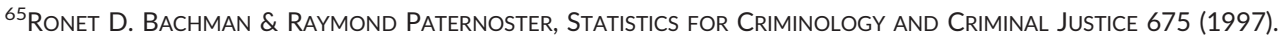

${ }^{66}$ JAMES JACCARD, INTERACTION EFFECTS IN LOGISTIC REGRESSION 12 (2001).

${ }^{67}$ Jennifer L. Skeem \& Christopher T. Lowenkamp, Race, Risk, and Recidivism: Predictive Bias and Disparate Impact, 54 CRIMINOLOGY 680, 692 (2016).

${ }^{68}$ Adam W. Meade \& Michael Fetzer, Test Bias, Differential Prediction, and a Revised Approach for Determining the Suitability of a Predictor in a Selection Context, 12 ORG. RES. METHODS 738, 740 (2009).

${ }^{69}$ Cecil R. Reynolds \& Lisa A. Suzuki, Bias in Psychological Assessment: An Empirical Review and Recommendations, in HANDBOOK OF PSYCHOLOGY 82, 101 (Irving B. Weiner ed., 2003).

${ }^{70}$ Meade \& Fetzer, supra note 68, at 741; Andrew D. Selbst, Disparate Impact in Big Data Policing, 52 GA. L. REV. 109. 121-22 (2017).

${ }^{71}$ Skeem et al., supra note 35 , at 585 .

${ }^{72}$ This result is consistent with logistic regression conducted by another team using a smaller subset of the Broward County dataset. Anthony W. Flores et al., False Positives, False Negatives, and False Analyses: A Rejoinder to "Machine Bias," 80 FED. PROBATION 38, 43 tbl. 5 (2016) These researchers did not discuss the gender variable or its indication of bias, presumably as they were focused on challenging ProPublica's conclusion about racial bias. 
${ }^{73}$ Results on file with author.

${ }^{74}$ Richard Berk, Accuracy and Fairness for Juvenile Justice Risks Assessments 16 J. Empirical Leg. Stud. 175, 191 (2019).

${ }^{75}$ Helmus \& Babchishin, supra note 59 , at 15.

${ }^{76}$ Chouldechova, supra note 57 , at 155.

${ }^{77}$ Berk, supra note 75, at 182.

${ }^{78}$ Dieterich et al., supra note 11 , at 33.

${ }^{79}$ Id.

${ }^{80}$ Jay P. Singh, Predictive Validity Performance Indicators in Violence Risk Assessment: A Methodological Primer, 31 BEHAV. SCI. \& L. 8,10 tbl. 1 (2013).

${ }^{81}$ See Flores et al., supra note 73, at 41 (suggesting both).

${ }^{82}$ Dieterich, supra note 11 , at 6.

${ }^{83}$ Singh, supra note 81 , at 15.

${ }^{84}$ Jay P. Singh et al., Measurement of Predictive Validity in Violence Risk Assessment Studies, 31 BEHAV. SCI. \& L. 55, 64 (2013).

${ }^{85}$ Martin Rettenberger et al., Prospective Actuarial Risk Assessment: A Comparison of Five Risk Assessment Instruments in Different Sexual Offender Subtypes, 54 INT'L J. Offender TherAPY \& COMP. CRIMINOLOGY 169, 176 (2010).

${ }^{86}$ Singh, supra note 81, at 16-18; Stephane M. Shepherd \& Danny Sullivan, Covert and Implicit Influences on the Interpretation of Violence Risk Instruments, 24 PSYCHIATRY, PSYCHOL. \& L. 292, 294 (2017).

${ }^{87}$ Singh, supra note 81 , at 16 .

${ }^{88}$ Id. at 17.

How to cite this article: Hamilton M. The sexist algorithm. Behav Sci Law. 2019;37:145-157. https://doi.org/ 10.1002/bsl.2406 